\title{
Educational kit for optical experiments
}

\section{Victor Dyomin, Igor Polovtsev}

Victor V. Dyomin, Igor G. Polovtsev, "Educational kit for optical experiments," Proc. SPIE 9665, Tenth International Topical Meeting on Education and Training in Optics and Photonics, 966512 (3 June 2007); doi: 10.1117/12.2207342

SDIE Event: Tenth International Topical Meeting on Education and Training in Optics and Photonics, 2007, Ottawa, Ontario, Canada 


\title{
Educational Kit for Optical Experiments
}

\author{
Victor V. Dyomin, Igor G. Polovtsev
}

Tomsk State University, 36 Lenin Avenue, Tomsk, 634050 Russia

+7(3822)412573 (tel./fax),dyomin@tsu.ru

\begin{abstract}
New educational kit and methodological instructions are presented in the paper. Methodological approaches and the kit design are improved and extended on the base of 10 years experience of the use of previous version of equipment. Basic approaches are the following: modular structure of the equipment with multifunctional blocks which are easily replaced; simplicity and unity of training equipment; diversity of light sources; possibility to rearrange the equipment and techniques both for universities and for high school; modified optical schemes for some experiments. The set of equipment includes: compact desk holographic installation, Hartle device, focal monochromator on the base of holographic lens, optical bench, prisms, lenses, gratings, collimator, several light sources (laser, LEDs, lamp), etc. Methodological instructions provided with this set include the list of demonstrations and laboratory works as well as links between various experiments and phenomena. Methodological instructions give recommendations for more than 50 demonstrations and practical works in various domains: light diffraction, interference of light, holography, geometric optics, Fourier optics, polarization effects, optics of spectrums, fiber optics, as well as a combination of the effects.
\end{abstract}

\section{Introduction.}

In comparison with the previous version of the kit [1], this equipment (UMOG-3) is more functional and provides more demonstrations and laboratory works. At the same time, the main principles of equipment design are the same - modular construction with the possibility to combine, rearrange and supplement the main blocks and elements; absence of complicated, precision and expensive units.

The kit allows realizing the number of demonstrations, including the following:

- edge diffraction;

- slit diffraction;

- hole diffraction;

- Young experiment;

- diffraction on a periodic structure;

- wedge interference;

- Newton rings;

- Lloyd mirror;

- polarization effects;

- Fourier-optics;

- phase contrast;

- observation of paths of reflected and refracted rays;

The kit allows realizing the number of training labs, including the following:

- recording of Denisyuk holograms;

- recording of double-exposed holographic interferogram;

- measuring of plate deformation by holographic interferometry method;

- measuring of distance between 2 holes on Young

pattern;

- measuring of diffraction grating period;

- Brewster angle measuring;

- measuring of prism internal reflectance angle and

refractive index;

- Fresnel formulae;

- Malus law studying;
- total internal reflection;

- light propagation through inhomogeneous mediums;

- optical heterogeneity visualization by shadow method;

- modeling of the light propagation through the fiber;

- demonstration of aberrations (spherical aberration, coma, astigmatism, distortion);

- white light polychromatic structure. 


\section{Set of equipment}

The overall view of basic set is given in fig. 1 .

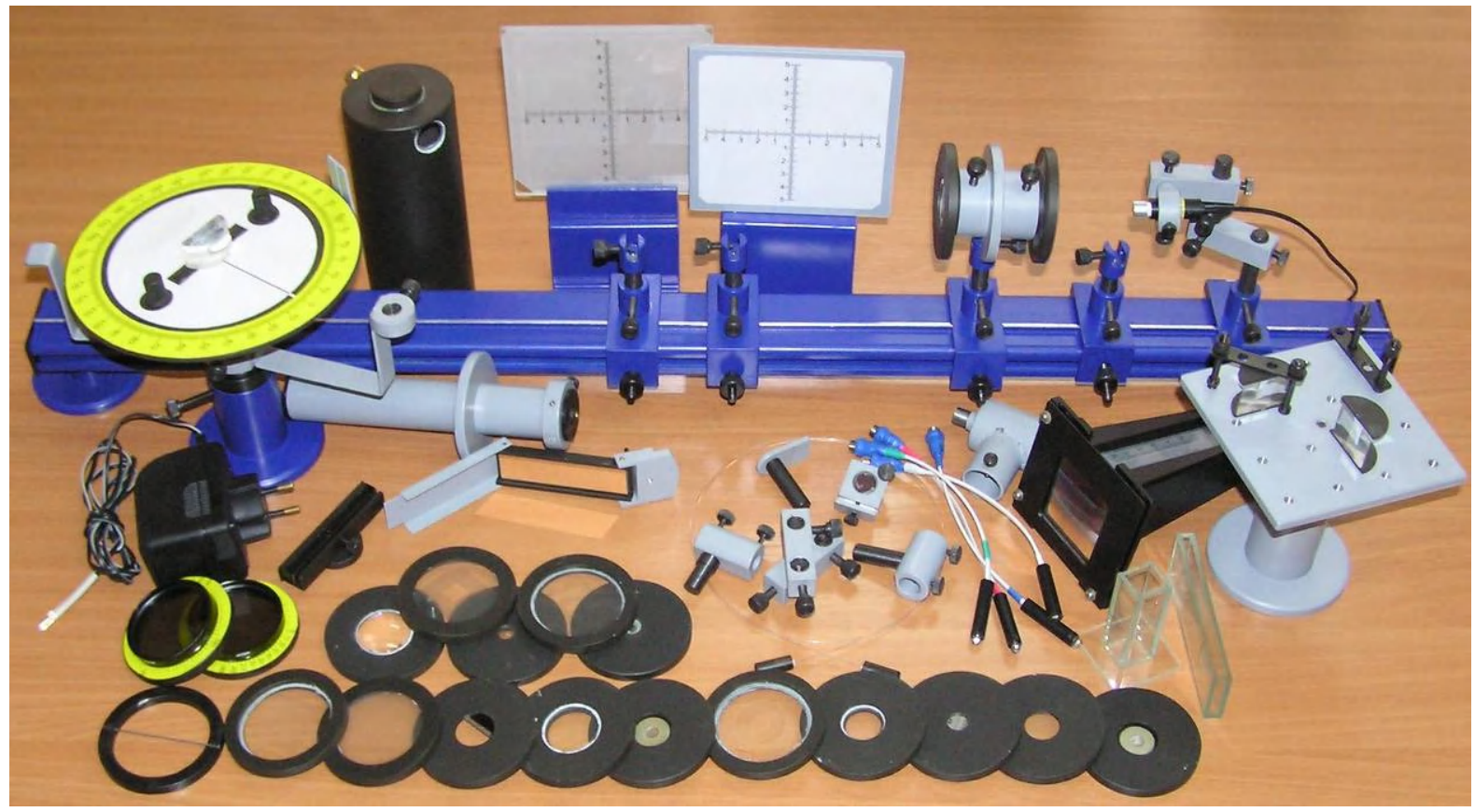

Fig. 1. The base set of equipment

UMOG-3 is performed as a set of functional assemblies compatible constructively and ideologically with each other: optical bench, Hartle device, desk holographic camera, focal monochromator, Lloyd mirror, projecting microscope, light beam expander, cylinder reservoir with illuminators.

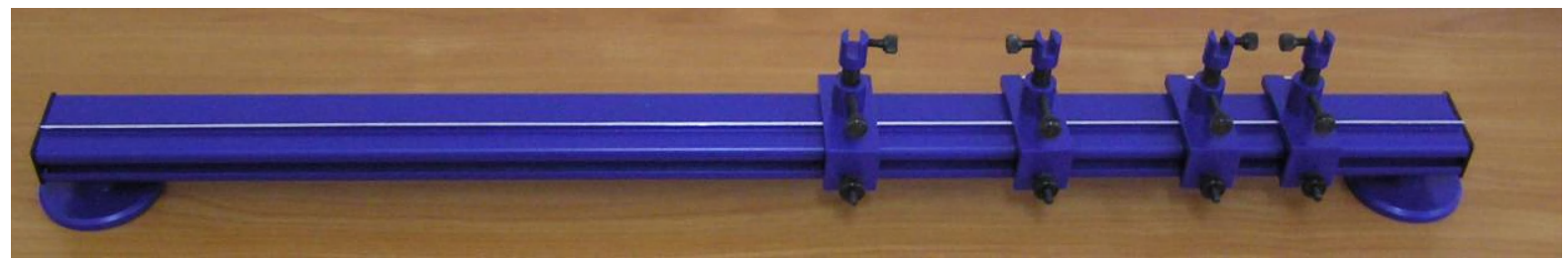

Fig. 2. Optical bench.

Optical bench (fig.2) is intended for breadboarding of experiment schemes and implemented in the form of guide where riders are fixed. The bench is mounted horizontally and has a ruler for riders' position measuring. In riders there can be mounted optical elements, adjusting devices, light sources, etc. In the kit the possibilities are foreseen both to mount optical elements on the axis of the bench and out of it.

Hartle device (fig.3) is meant for experiments of light refraction and fiber optics.

Desk holographic camera is meant for Denisyuk hologram registration and it is described in detail in [1]. 


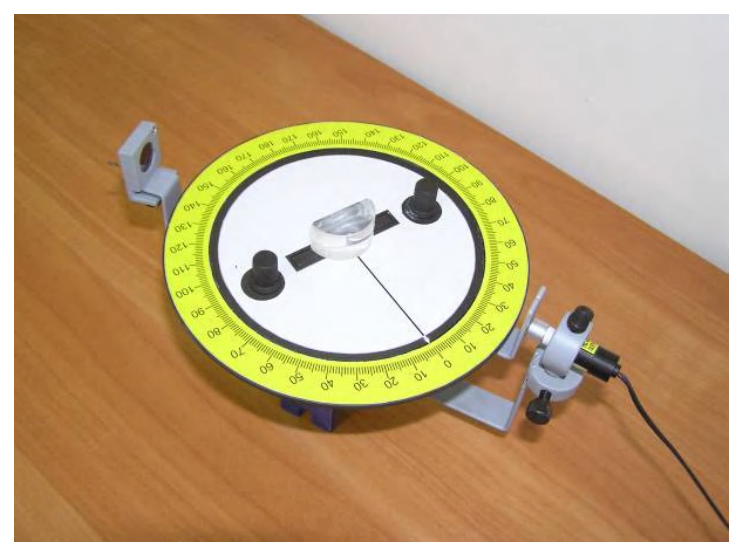

Fig. 3. Hartle device as a unit for geometrical optics experiments

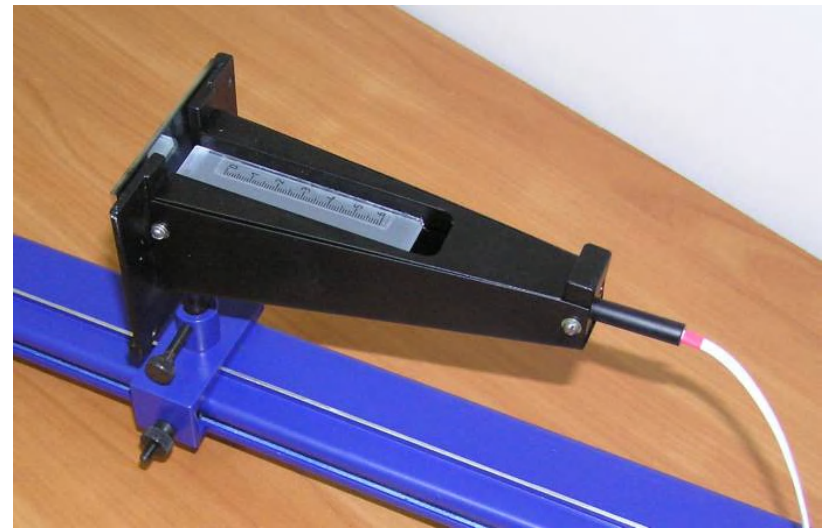

Fig. 4. Focal monochromator on a base of holographic lens.

Focal monochromator with holographic lens (fig. 4) is meant for the spectrum optics experiments and for spectral measurements. Spectrum is formed on the translucent screen with the measuring grid instead of that the CCD-censor can be mounted. Light from the light sources is introduced into monochromator by the optical fiber or from the LED directly.

Lloyd mirror (fig. 5) is meant for producing of two coherent sources for interference studying.

Horizontal projecting microscope (fig. 6) is meant for observation of enlarged by the microobjective images of objects on the translucent screen with the measuring greed. Instead of the screen a CCD-array can be mounted. The microobjective of the microscope is also used in holographic camera for beam expansion.

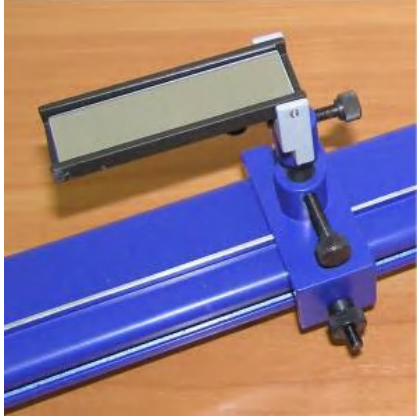

Fig. 5. Lloyd mirror.

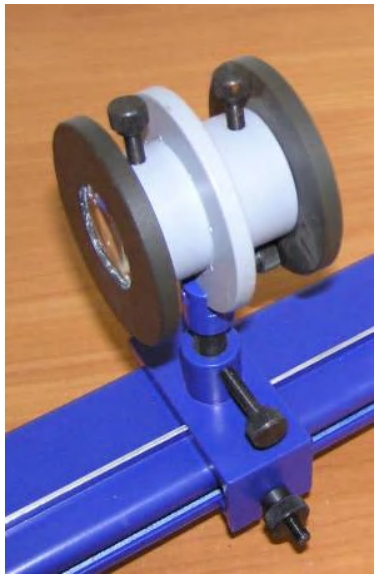

Fig. 7. Light beam expander (collimator)



Fig. 8. Cylinder reservoir with illuminators.



Fig. 6. Horizontal projecting microscope.

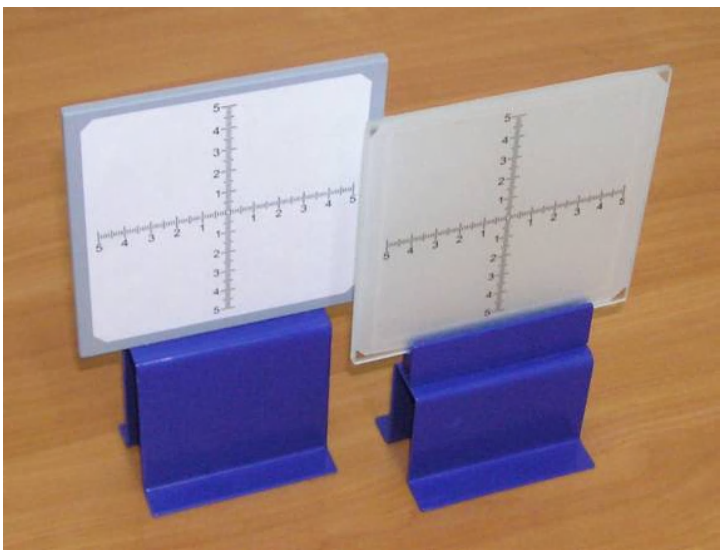

Fig. 9. Screens. 
Light beam expander (fig. 7) is meant for parallel beam forming with $25 \mathrm{~mm}$ diameter. By the expander it is possible to form divergent (convergent) beam. Optical scheme (Galilean telescope) consists of microobjective and objective, changing distance between them one can obtain parallel or required divergent beam.

Cylinder reservoir with illuminators (fig. 8) is meant for "lighting stream" demonstration.

Screens (fig. 9) are meant for observation of images of objects, diffraction and interference patterns, etc., in transmitted or reflected light. For visual measuring the screens are supplied by grids.

Various light sources are included into kit: metal-halide lamp, laser semiconductor diode ( $\lambda=655 \mathrm{~nm})$; light emitting diodes - red $(\lambda=630-632 \mathrm{~nm})$, blue $(\lambda=471-475 \mathrm{~nm})$, green $(\lambda=520-530 \mathrm{~nm})$, white $(\lambda=632,520,473$ $\mathrm{nm})$. In holographic camera it is used He-Ne laser $(\lambda=633 \mathrm{~nm})$. Spectral characteristics of light sources are given in fig. 10.

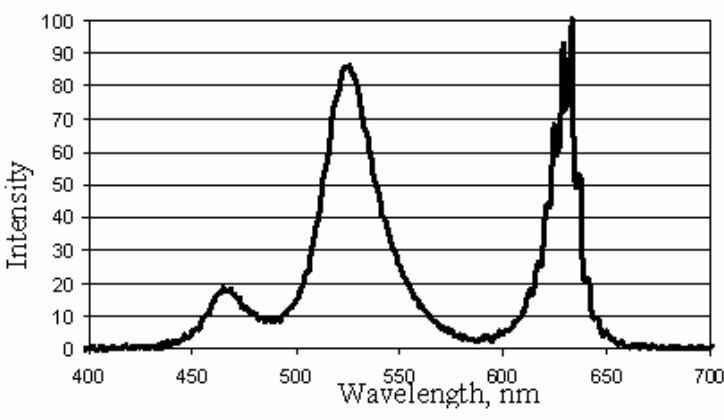

a) white LED

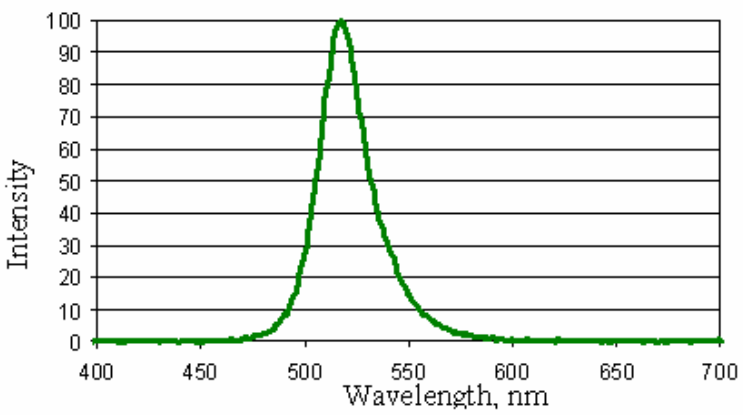

c) green LED

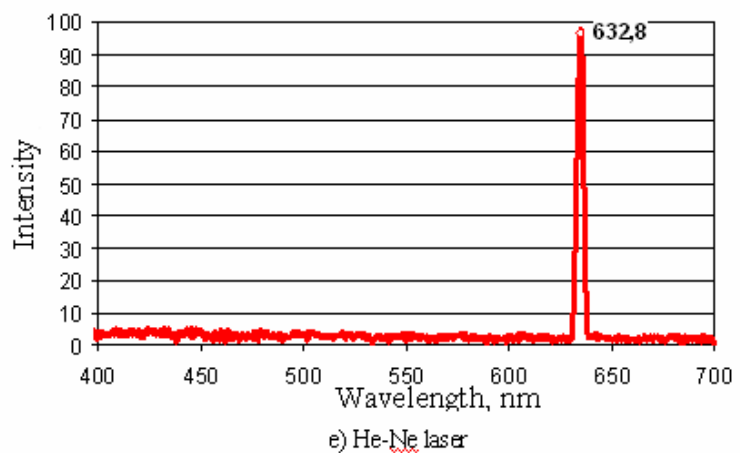

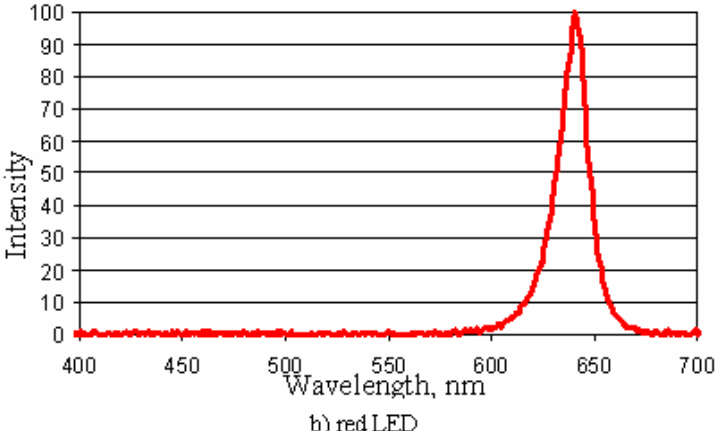

b) red LED

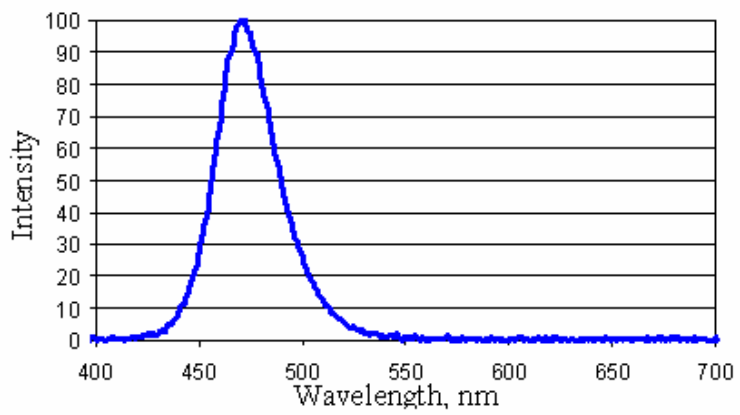

d) blue LED



f) semiconductor laser

Fig. 10. Spectral characteristics of light sources.

The kit also includes a set of optical elements (fig. 1) with holes, diffraction gratings, lenses, prisms, fibers, etc.

\section{Methodological recommendations}

The primary methodological recommendations for experiments in diffraction, interference, Fourier-optics, geometrical optics, holography are described in work [1]. The examples of observed patterns are shown in fig. 11. In this paper we give methodological approaches just for some additional experiments realized by new kit. 




Slit diffraction



Young pattern

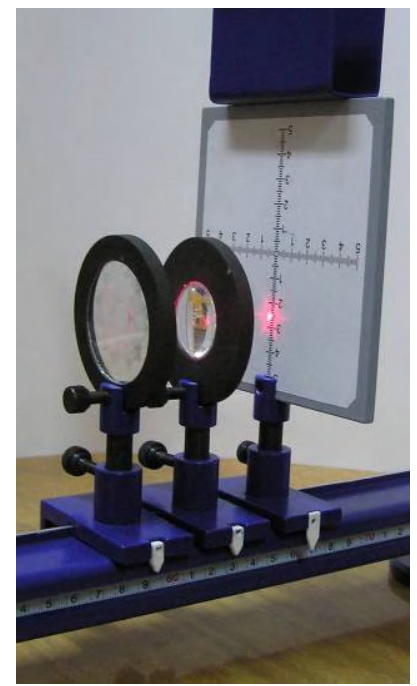

Fourier-spectrum observation

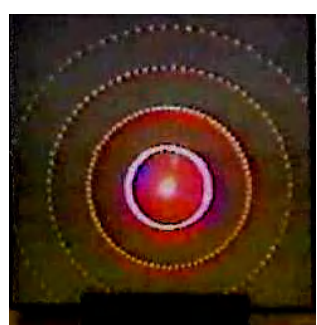

Diffraction on a circular grating

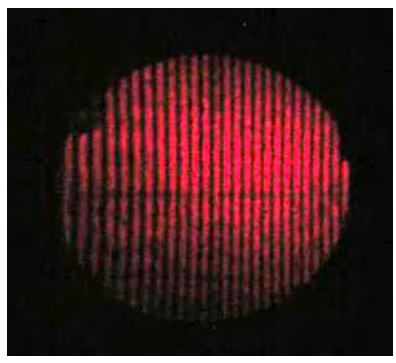

Wedge interference

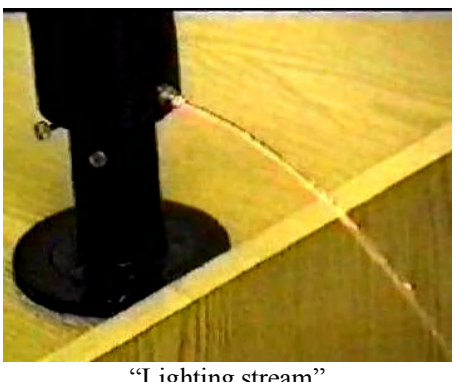

"Lighting stream"



Diffraction on crossed gratings

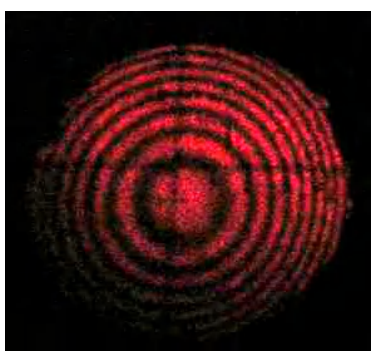

Newton rings

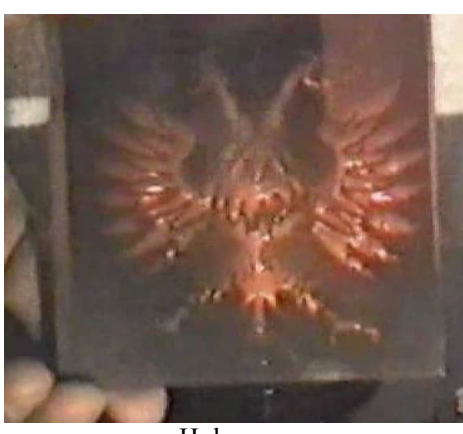

Hologram

Fig. 11. Examples of patterns observed by using UMOG-3.

\subsection{Measuring of a positive lens focal length.}

In the previous methodical recommendations two methods of focal distance measuring were examined - by using diffraction grating and in experiment with Newton rings. In this version new experiment is added - with the use of wedge. The experiment is based on using of horizontal microscope (fig. 6) for focal spot observation.

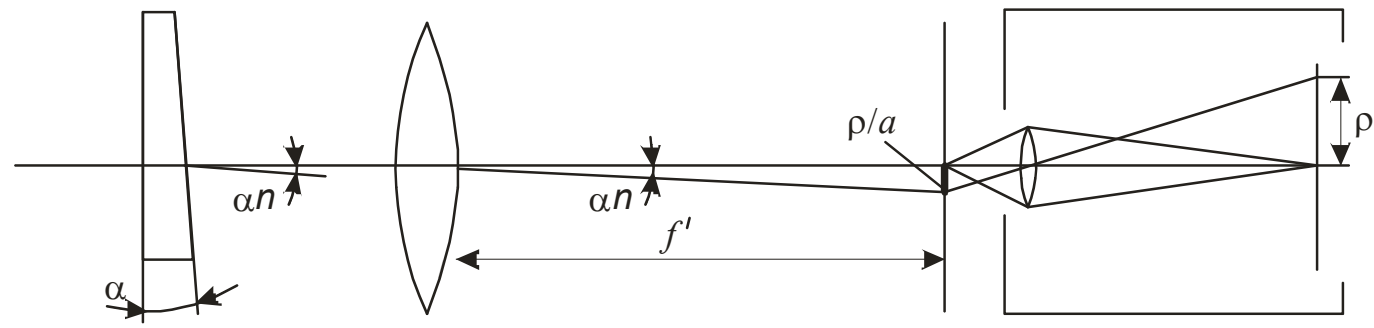

Fig. 12. Focal distance measuring using wedge. 
Placing the wedge before lens and revolving it around the optical axis one can observe beating of focal spot on the microscope screen (fig. 12). Screen grid allows measuring circle radius $\rho$ delineated by the spot. Then by known values of wedge angle $\alpha$ evaluated beforehand in technique [1], as well as microscope magnification $a$, one can define lens focal distance using formula $f^{\prime}=\frac{\rho / a}{\alpha \cdot n}$, where $n=1.52$ - refractive index of wedge material. Here the fact is used that sine and tangent of small angle (that are $\alpha$ and $\alpha n$ ) are equal to this angle.

For microscope magnification definition one can use one of diffraction elements with known period included in the optical elements set.

\subsection{Fiber optics}

\subsubsection{Hartle device experiments}

Modified Hartle device, as in previous kit, allows studying the internal reflectance effect, Brewster angle measuring, Fresnel formulae studying (fig. 13, 14). Placing diffraction grating with measured beforehand period, in the middle of the Hartle device disk (instead of half-cylinder prism), one can realize the experiment for measuring by photodiode of intensities of various diffraction peaks. Obtained data are used for grating profile evaluations.

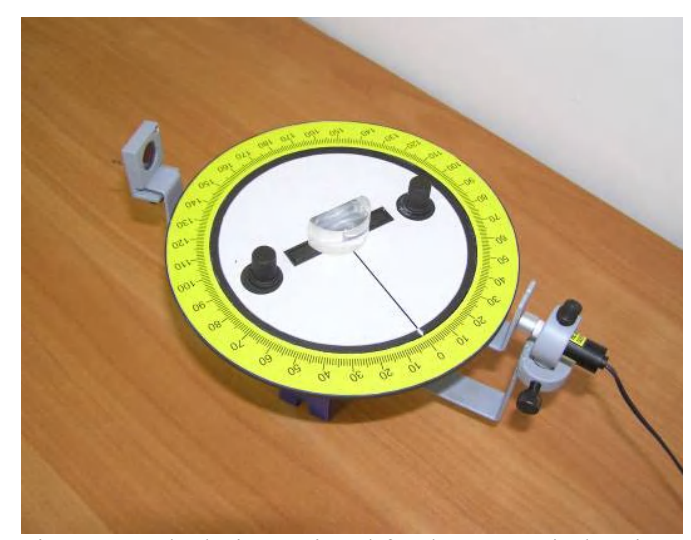

Fig. 13. Hartle device equipped for the geometrical optics demonstrations.



Fig. 14. Snell law demonstration.

In the realized version of UMOG-3 Hartle device is also used for fiber loss measurement that are caused by fibers angle misalignment. This effect is studied by two macro-models of light guides (fig. 15) - glass rods. The loss appears while disk revolving with fixed light guide within the limits provided by air-gap. Loss value is measured by the photodiode, angle of misalignment - by the scale of Hartle device.

\subsubsection{Measuring of fiber loss caused by air gap between the fibers.}

This effect is also studied by using of 2 light guide macro-models. The light guides are situated by holders in alignment with butts closely (fig. 16). On other butts of the rods the semiconductor laser diode and photodiode are fixed. The loss appears when air gap between butts appears. The loss value is measured by photodiode, air gap - by optical bench scale or by caliper. 


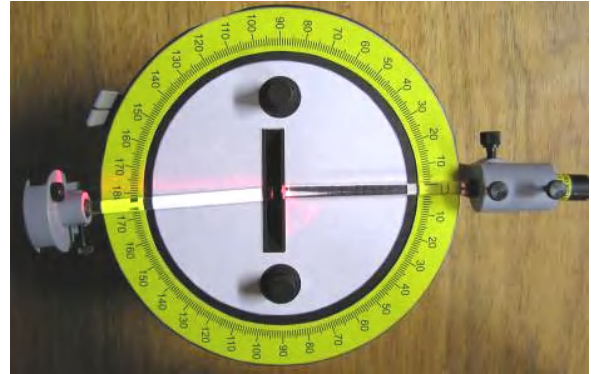

Fig. 15. Fiber loss studying while misalignment of models of fiber optics elements.

\subsubsection{Loss caused by optic fiber bending.}

When fiber is bending, the total internal reflectance law is not fulfilled for higher order modes. So light does not reflect from the cover but spreads in it and so is absorbed. Theoretical loss defined by the following method: $\alpha=-10 \lg [1-a /(R \Delta)]$, where $a$ - core diameter, $R$ - bend radius, $\Delta=0,01$. Evaluation of the loss depending on fiber bend is made by scheme on fig. 17. For that it is used optical fiber, photodiode, one of light sources, proper holders from UMOG-3 kit as well as disks of various radiuses cut from fiberboard and fixed on plateau from UMOG-3 kit.

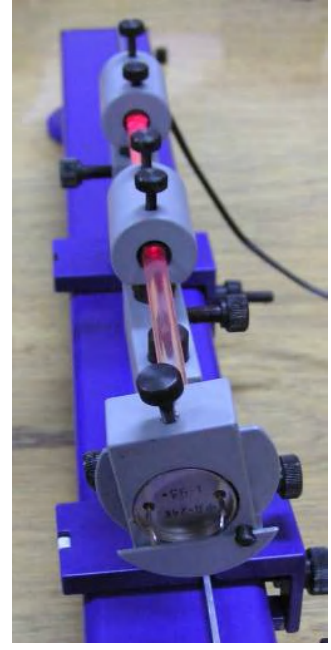

Fig. 16. Studying the loss caused by air gap between elements.

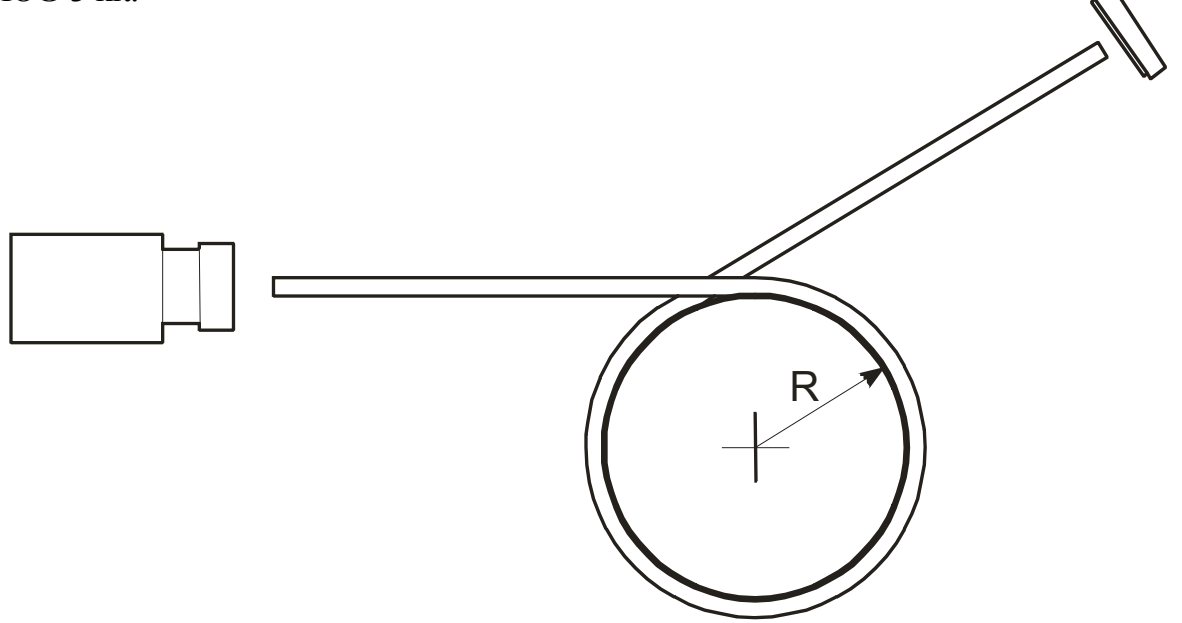

Fig. 17. Scheme for the fiber loss measuring depending on optical fiber bend.

During the work optical fiber is bent by turns to different radiuses and it is simultaneously measured received intensity. Knowing dissipation index for different disk radiuses one can for example determine fiber core diameter using the formula given above.

\subsubsection{Fiber sensor of longitudinal shift.}

The equivalent scheme of radiation putting into fiber is given in fig. 18. For that the incoherent light source 5.H and the objective 4.2.2 are fixed on the optical bench. Focal point is projected on the fiber butt 4.3.3. On another light guide butt the photodiode 6.1 is fixed. The efficiency of radiation putting in depends on the butt of light guide location relative to the focus. This dependence can be used for construction of shift sensor. In this work it is supposed to formulate this dependence. The photodiode here is used for photo-current measuring, for shift measuring one can use the caliper. 


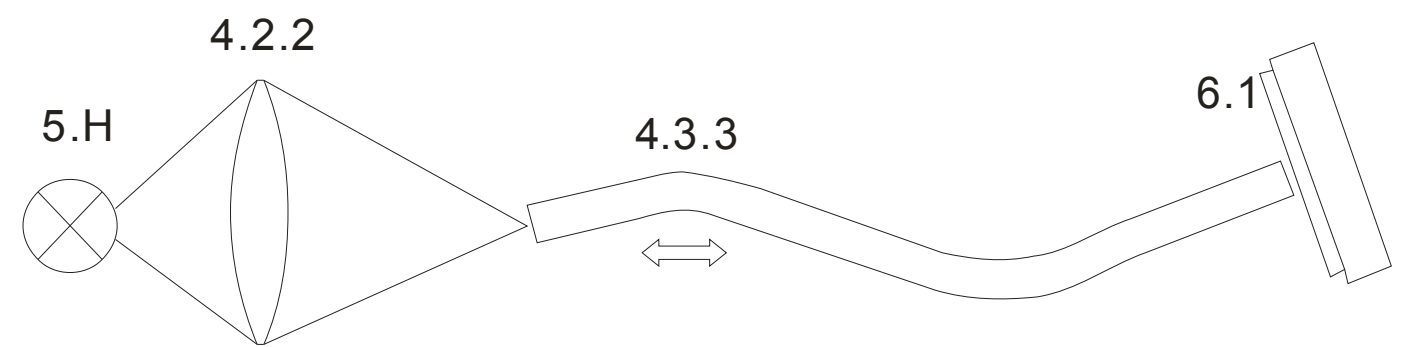

Fig. 18. Longitudinal shift sensor scheme.

It is clear that using of this scheme for real sensor construction is not expedient but it is possible. Here it is suggested for only methodical view.

More acceptable example is light guide sensor scheme (also realized using UMOG-3 kit) where light guide input butt is fixed and used for receiving of radiation scattered by moving surface. Surface lighting is executed by laser semiconductor diode. Luminous flux transmitted onto photodetector 6.1 over the fiber 4.3.3 depends on distance between the surface and input butt that is used in technical solution.

\subsection{Optical spectrum experiments.}

In the kit the possibility is provided to realize the traditional scheme of prism monochromator (fig. 19). The following items are fixed on the optical bench: incoherent light source 5.H, objective 1.7.O, slit 4.1.9, longfocus lens 4.2.1, and prism 4.2.4. Continuous spectrum of the source is observed in the plane of slit image on the translucent screen 6.4.P. For more effect one can use double monochromatization by the second prism.

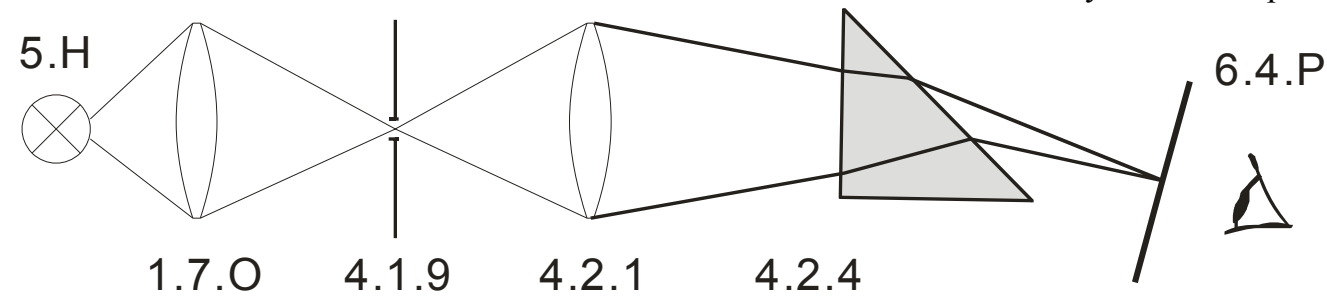

Fig. 19. Prism monochromator scheme.

At the same time it is useful for studying process to use focal monochromator on basis of holographic lens. While using one it is necessary to become proficient in most domains of interference, diffraction, holography, spectrum optics, etc. Special refinement is added to these experiments by using the same holographic optical element as dispersive and focusing unit.

\subsubsection{Holographic lens theory.}

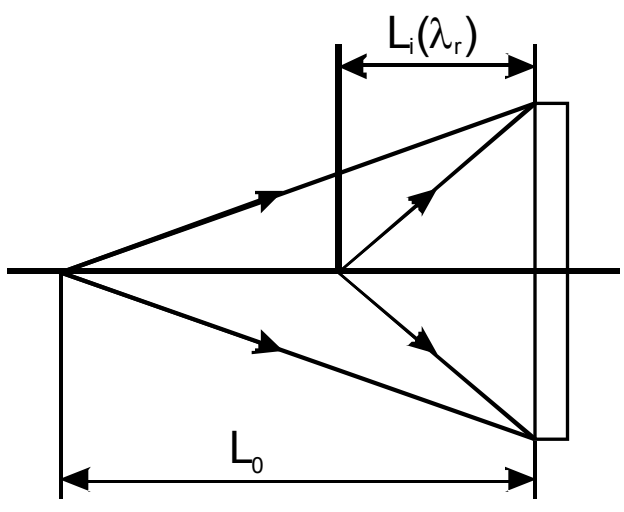

a

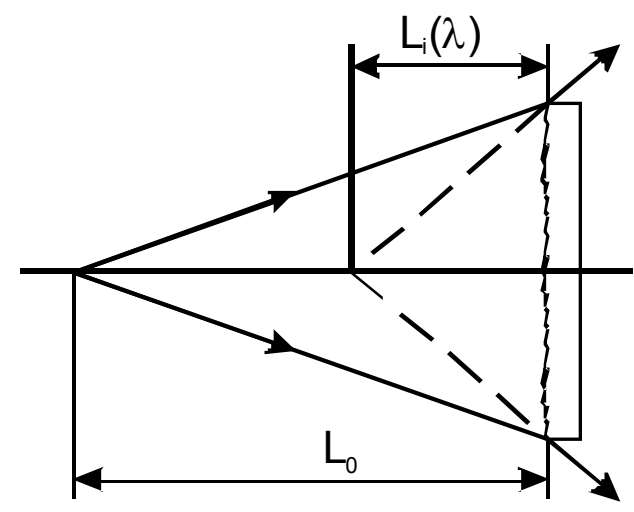

b

Fig. 20. Recording (a) and reconstruction (b) of holographic lens. 
If one records on photographic plate an interference pattern from two spherical waves with wavelength $\lambda_{\mathrm{r}}$ and with centers of curvature of wave fronts on distances $L_{o}$ and $L_{i}\left(\lambda_{r}\right)$ (fig. 20a), then after photographic development it will be planar hologram that possesses lens properties. If such hologram being illuminated with spherical wave (fig. 20b) the restored wave front is also spherical. Focal length of such lens is described by the formula:

$$
\frac{1}{f^{\prime}}=\frac{1}{L_{0}}+\frac{1}{\mathrm{~L}_{\mathrm{i}}\left(\lambda_{r}\right)}
$$

When holographic lens is illuminated by reference radiation with wavelength $\lambda$ from distance $L_{o}$, the image is observed on distance $L_{i}(\lambda)$ associated with registration parameters by equation:

$$
L_{i}(\lambda)=\lambda_{r} \frac{L_{i}\left(\lambda_{r}\right) \cdot L_{0}}{L_{i}(\lambda) \cdot\left(\lambda-\lambda_{r}\right)-\lambda \cdot L_{0}} \approx \frac{\lambda_{r}}{\lambda} \cdot L_{i}\left(\lambda_{r}\right)
$$

Here it is supposed that $(\lambda-\lambda)<\lambda$ and $L_{i}<L_{0}$.

Hence it follows that holographic lens possesses pronounced chromatism that can be used in design of spectral devices. Monochromator schemes utilizing dependence of focal distance from wavelength are known for a long time and are called as focal monochromators.

\subsubsection{Focal monochromator with holographic lens (FMHL).}

The FMGL scheme is given in fig. 21. If one makes chemical treatment of photo-plate so that to obtain relief-phase hologram and then apply mirror coating by vacuum deposition over the obtained relief, then it will be reflective dispersive element of focal monochromator with holographic lens that we will denote as HL. The $\mathrm{HL}$ is illuminated by light source with wavelength $\lambda$ from distance $L_{0}$. Reconstructed image is observed from distance $L_{i}(\lambda)$ described by equation (2). Using in scheme the off-axis fragment of HL allows forming separately in space images formed by different wavelengths. Images in described here construction are observed on translucent screen with measuring grid (fig. 4).

Denote $\mathrm{y}_{0}$ - size of light source, $\mathrm{h}_{0}$ - coordinate of dispersive element point from that HL edge. The HL forms the image with size $y=\beta \times y_{0}$ where $\beta$ - linear magnification coefficient of HL that is connected with recording - reconstruction parameters:

$$
\beta=\frac{\mathrm{L}_{\mathrm{i}}(\lambda)}{L_{0}}
$$

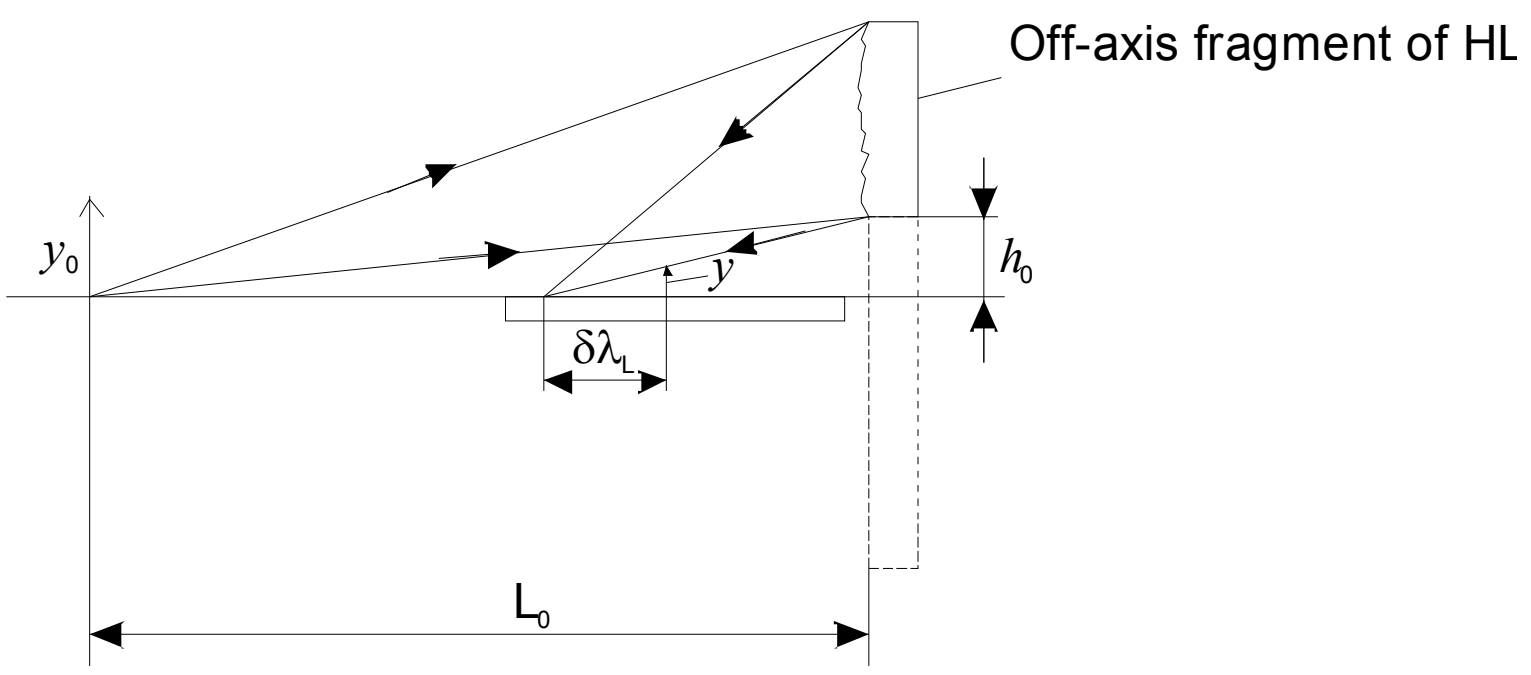

Fig. 21. Optical scheme of FMHL. 
As long as on the translucent glass it is observed the projection of image of size $y$ on optical axis then for its size $\delta \lambda_{L}$ we have evident equation:

$$
\delta \lambda_{L}=\frac{y \cdot L_{i}(\lambda)}{h_{0}-y}=\frac{L_{i}^{2}(\lambda) \cdot y_{0}}{L_{0} \cdot\left(h_{0}-\beta \cdot y_{0}\right)} \approx \frac{L_{i}^{2}(\lambda)}{L_{0}} \cdot \frac{y_{0}}{h_{0}}
$$

Then as spectral resolution of the focal monochromator with off-axis fragment of HL we may take value

$$
\delta \lambda=\delta \lambda_{\mathrm{L}} \cdot \frac{\Delta \lambda}{\Delta \lambda_{\mathrm{L}}}
$$

where $\Delta \lambda_{L}=L_{i}\left(\lambda_{S}\right)-L_{i}\left(\lambda_{L}\right)$ - area occupied on translucent screen of monochromator by spectral range $\Delta \lambda$ with shortwave limit $-\lambda_{S}$ and longwave $-\lambda_{L}$.

The HL used in monochromator UMOG-3 has the following parameters

$$
L_{o}=150 \mathrm{~mm} ; \quad L_{i}\left(\lambda_{r}\right)=40 \mathrm{~mm} ; \quad \lambda_{r}=632,8 \mathrm{~nm} ; \quad h_{0}=10 \mathrm{~mm} .
$$

If optic fiber is used as light source then for $y_{0}$ it should be taken the diameter of fiber core equals $0.25 \mathrm{~mm}$. Thus it is possible to bring light from different light sources from UMOG-3 kit into focal monochromator. The spectral resolution estimated by (5) is about $50 \mathrm{~nm}$. This value can be improved by screening a part of HL.

Instead of the translucent screen it can be used CCD sensor. It allows observing formed spectrum in monitor and make proper measuring with digital signal.

\subsubsection{Spectral measuring with FMHL.}

Relief-phase grating (HL) used in UMOG-3 is non-linear in principle. Thereby while diffraction on such structure there are observed several diffraction orders. It should be remembered while spectrum interpretation obtained by FMHL and try to make experiments at the same order. The higher diffraction order is, the bigger angle the beam deflects while interaction with the grating. Hence the more number of order is, the closer it is situated to HL.

White light polychromatic structure is observed on the translucent screen of monochromator while the light is used from halogen lamp or white LED. White LED consists of 3 crystals generating radiation of 3 wavelengths (linear spectrum). Therefore their images are seen separately in spectrum (fig. 22b) as against thermal source spectrum (fig. 22a) - metal-halide lamp - that is principally continuous.
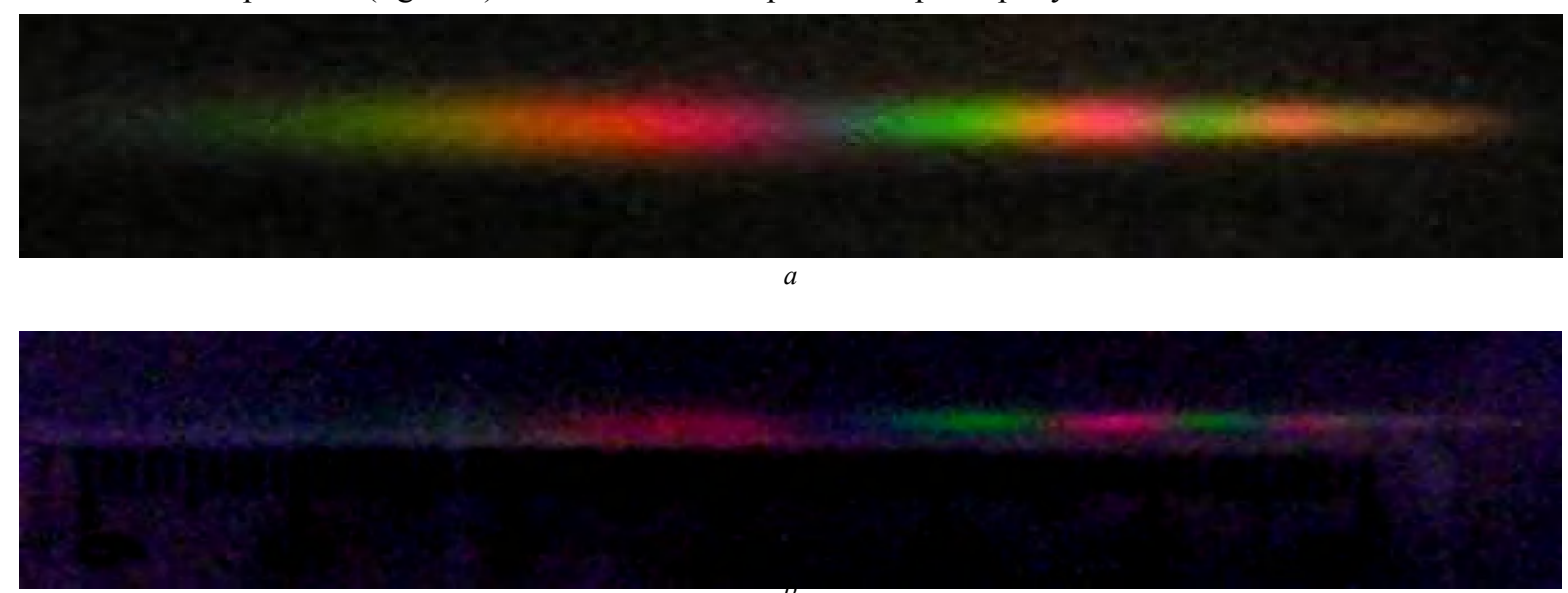

Fig. 22. Different white light sources spectrums: a-metal-halide lamp, b- white LED. 
In order to measure the wavelength of monochromatic source it is necessary to calibrate the device. From (2) it follows that

$$
L_{i}(\lambda)=\frac{k}{\lambda}
$$

where $k$ is the constant depending on registration parameters.

Therefore using in FMHL the radiation from source with known wavelength $\lambda_{0}$ (for example from laser diode) and measuring image location $L_{i}\left(\lambda_{0}\right)$ on the translucent screen one can calculate for given monochromator and given diffraction order

$$
k=L_{i}\left(\lambda_{0}\right) \cdot \lambda_{0}
$$

For measuring $L_{u}(\lambda)$ one can use monochromator screen grid. Better results can be obtained by means of caliper.

After that one can introduce into monochromator the radiation from source with unknown wavelength and having measured $L_{i}\left(\lambda_{U}\right)$ obtain for its wavelength

$$
\lambda_{U}=\frac{L_{i}\left(\lambda_{0}\right)}{L_{i}\left(\lambda_{U}\right)} \cdot \lambda_{0}
$$

\section{Conclusion}

In this paper the new version is described of the educational kit for optical experiments. It is shown that the new version (UMOG-3) is more functional and provides more demonstrations and laboratory works. At the same time, the main principles of equipment design remain valid - modular construction with the possibility to combine, rearrange and supplement the main blocks and elements; absence of complicated, precision and expensive units. This approach to the design of such a set provides a possibility of its rearrangements, when necessary, to satisfy the needs of teaching process either for universities or for high schools. Moreover such methodical approaches and designs allow simplifying and unifying training equipment in optics and give an opportunity to concentrate an attention on phenomenon researched instead of on instrumental provision.

In the paper the methodological approaches are presented just for some additional experiments realized with the use of new kit: fiber optics experiments, spectrum experiments, spectral measurement.

References

1. V.V. Dyomin, I.G. Polovtsev, "Set of instrumentation and methodological instructions for practical work in optics", SPIE Proceedings, 2525, P. 45 - 55 (1995). 